\title{
La opresión del género sobre las trayectorias de vida de mujeres alcohólicas $^{1}$
}

\author{
Francisca Colimil ${ }^{2}$ y Daniela Paz Ramírez ${ }^{3}$
}

\author{
Fecha de recepción: 01 de noviembre de 2016 \\ Fecha de aprobación: 07 de diciembre de 2016
}

\begin{abstract}
Resumen
Los datos de Senda (2016) dan cuenta del incremento del consumo de alcohol en Chile en los últimos años; pese a lo anterior, la mayoría de la información levantada sobre el tema se ha realizado desde una perspectiva cuantitativa y sin enfoque de género. Por ello, la presente investigación aborda elementos provenientes de la cultura heteronormativa, comunes a las trayectorias de consumo de alcohol en mujeres alcohólicas, y cómo estos se manifiestan de forma transversal en la vida de estas mujeres, independientemente de su nivel socioeconómico y de estudios. Es decir, desde el supuesto de que ser mujer y alcohólica no tiene las mismas implicancias sociales que ser hombre y alcohólico, en tanto que la opresión normativa del género -y como consecuencia el estigma- recae con mayor fuerza sobre las mujeres por contravenir el "deber ser" mujer. Esta investigación, desde un enfoque cualitativo y con el método biográfico, se propone explorar cómo dichos elementos se hacen presentes en sus relatos de vida.
\end{abstract}

Palabras clave: cultura patriarcal, género, violencia, alcohol, estigma.

\section{The gender oppression on the life trajectories of alcoholic women}

\begin{abstract}
The data of Senda (2016) account the increase of the alcohol consumption in Chile, in the last years; however, most of the information gathered on this subject have been conducted from a quantitative perspective, and without a gender approach. Because of this, the present research addresses elements derived from the heteronormative culture, common to the alcohol consumption of alcoholic women, and how do they express transversally on the life of these women, regardless of their educational and socioeconomic status. In other words, from the assumption that being woman and alcoholic doesn't have the same social implications tan being man and alcoholic, whilst the normative
\end{abstract}

Este artículo deriva de la tesis realizada para optar al título de Licenciadas en Sociología durante el año 2016, en la Universidad Católica Silva Henríquez.

Socióloga, Universidad Católica Silva Henríquez. Contacto: fca.colimil@gmail.com

Socióloga, Universidad Católica Silva Henríquez. Contacto: dpazrt@gmail.com 
gender oppression -and therefore, the stigma- falls with a higher strength on women, because of infringing the "ought-to-be" woman. This investigation, from a qualitative approach y with the biographical method, proposes the exploration of how these elements take place in their life stories.

Keywords: patriarchal culture, gender, violence, alcohol, stigma.

\title{
A opressão do gênero nas trajetórias de vida de mulheres alcoólatras
}

\begin{abstract}
Resumo
Os dados de SENDA (2016) dão conta do aumento do consumo de álcool no Chile nos últimos anos; apesar disso, a maioria das informações sobre a questão levantada, foi feita a partir de uma perspectiva quantitativa e sem o enfoque do gênero. Portanto, esta pesquisa aborda elementos derivados da cultura heteronormativa, comuns as trajetórias de consumo de álcool em mulheres alcoólicas, e como estes se manifestam transversalmente nas vidas dessas mulheres, independentemente de sua condição socioeconômica e educação. Ou seja, a partir do pressuposto de que ser mulher e alcoólatra não tem as mesmas implicações sociais que ser um homem e alcoólatra, enquanto que a opressão normativa do gênero -como resultado do estigma- cai mais fortemente sobre as mulheres porque viola o "dever ser" mulher. Esta pesquisa, a partir de uma abordagem qualitativa e com o método biográfico, tem como objetivo explorar como esses elementos estão presentes em suas histórias de vida.
\end{abstract}

Palavras-chave: cultura patriarcal, género, violência, álcool, estigma

\section{Introducción}

En el mundo, 29 millones de personas padecen trastornos relacionados con las drogas y el alcohol. En Chile, según los datos del Senda (2016), el incremento del consumo de alcohol alcanzó en 2014 un 79,1\% en la población general, mientras que el consumo en mujeres llegó al 64,9\% en 2012; es decir más de la mitad de la población de mujeres encuestadas consume alcohol (Senda, 2016).

A partir de lo anterior, la investigación surgió del supuesto de que ser mujer y alcohólica no tiene las mismas implicancias sociales que ser hombre y alcohólico, en tanto que la opresión normativa del género -y como consecuencia, el estigma- recae con mayor fuerza sobre las mujeres, fundamentalmente por contravenir o no responder a las expectativas sociales del "deber ser" mujer, produciéndose entonces acciones evasivas de la realidad, una de ellas es el consumo problemático de alcohol. 
Esta investigación se propuso conocer trayectorias de vida de mujeres alcohólicas, para comprender de qué manera operaron los elementos provenientes de la sociedad heteronormativa ${ }^{4}$, los procesos de socialización y las diferentes vivencias que se desarrollan en una cultura marcada por la dominación masculina en las historias de vida de estas mujeres, considerando sus inicios en el consumo de alcohol, el desarrollo de su alcoholismo y su posterior recuperación.

Cabe destacar la escasez tanto de investigaciones cualitativas que estudien los casos particulares de dicho fenómeno, como también con enfoque de género.

Es preciso entender el alcoholismo de mujeres como un fenómeno multidimensional, puesto que conlleva una serie de problemas sociales asociados a la violencia hacia la mujer en sus distintas expresiones, además de ser historias marcadas por la estigmatización y rechazo.

En primer lugar, este artículo entregará antecedentes del problema de investigación; en segundo término apoyará los antecedentes con una revisión bibliográfica acerca del tema; luego se planteará la metodología de la investigación para dar paso, en cuarto lugar, a los resultados y, finalmente, aportar las conclusiones de la investigación.

\section{El problema del alcoholismo en mujeres desde la perspectiva de género}

El discurso patriarcal dominante en la sociedad señala que las mujeres cumplen un rol histórico al encargarse de los asuntos domésticos, con el objetivo de, por un lado, llevar a cabo la crianza exitosa de los hijos y, por otro, encargarse del mantenimiento

\footnotetext{
4 Entendiendo por esto, no solo el "deber ser" respecto de la heterosexualidad y los roles reproductivos, sino también la "construcción social asimétrica relacional entre los géneros leídos binariamente" (Cáceres, 2016, p. 246).
} 
de la armonía en el hogar, para que los hombres se desempeñen de manera óptima en su trabajo (Colimil y Ramírez, 2016).

Estudiar la problemática desde la perspectiva de género en el consumo de alcohol resulta importante, pues los roles y la opresión que socialmente se le asigna al género femenino en algunas ocasiones genera acercamiento al consumo de alcohol y, a su vez, si dicho consumo es problemático, es considerado socialmente como una conducta desviada y en consecuencia estigmatizadora, entre otras cosas porque el consumo de alcohol se contrapone a la actividades cotidianas asociadas al "rol femenino" en la sociedad patriarcal. Por ejemplo, las mujeres, tengan o no un trabajo remunerado, deben realizar labores domésticas, de educación y crianza de los hijos/as; en cambio en los hombres el consumo de alcohol es considerado una práctica propia del género, porque son ellos quienes proveen y sustentan la familia, y cuando finalizan sus jornadas laborales tienen socialmente permitido momentos de recreación con los amigos, desligándose de labores propias del hogar y del cuidado hacia los hijos. No obstante, cuando las mujeres solo se desenvuelven en el contexto doméstico, sufren algún tipo de violencia de género, experiencias de abandono $u$ otras; suponemos, por tanto, que dichas experiencias motivan a estas mujeres a realizar prácticas evasivas, y el consumo problemático de alcohol es una de éstas. Es necesario agregar que las experiencias asociadas al género no solo están presentes en los inicios del consumo, sino que de éstas también emerge una serie de situaciones relacionadas con la recuperación, aunque de manera ambivalente, tanto como presión para la recuperación como para el ocultamiento de la condición alcohólica.

Se trabajó con mujeres pertenecientes a Alcohólicos Anónimos (A.A.) ${ }^{5}$, puesto que, si bien era necesario conocer trayectorias de consumo de alcohol, en términos metodológicos era nece-

5 Organización creada en Estados Unidos en 1935, la que gracias a su efectividad en los tratamientos, adoptó un carácter internacional (Alcohólicos Anónimos, 2016). 
sario que estas mujeres estuvieran sobrias al momento de realizar el relato para que así pudieran reflexionar sobre su consumo.

Las personas pertenecientes a A.A. definen el alcoholismo como "una enfermedad progresiva, que no puede curarse pero que, igual que muchas enfermedades, puede contenerse" (Alcohólicos Anónimos, 2016). Suponen que hay una debilidad física y una obsesión por el alcohol, por eso es importante el apoyo del grupo para conseguir la abstinencia, poder recuperarse y efectivamente mantenerse alejado del alcohol para siempre.

\section{Algunos lineamientos teóricos para comprender el problema}

En Europa, a partir del año 1673, a raíz de la publicación del libro De l'égalité des sexes del filósofo François Poullain de la Barre, comenzó a tematizarse el origen de la subordinación de las mujeres, entendiendo que era un prejuicio presente en la educación impartida a mujeres y hombres en el marco de "una estructura de dominación masculina (...) responsable de una de las desigualdades medulares de la sociedad moderna" (Cobo, 2009, p. 14). Para Castells (2000) dicha desigualdad perdura hasta hoy, principalmente debido a la imposición de autoridad por parte de las instituciones en la unidad familiar, llevando el nombre de "patriarcado". Según Cáceres (2016), dicha estructura dominante "reproduce los patrones heteronormativos de la sociedad patriarcal" (p. 243), en los que "no solo existe una construcción social asimétrica relacional entre los géneros leídos binariamente sino que, además, tenemos un escenario en el que los cuerpos en términos de asimetría física refuerzan dicha relación" (p. 246) de subordinación femenina mediante la violencia.

En esta misma lógica, se educa a las niñas para que sean delicadas, femeninas y cuidadosas con su cuerpo (Beauvoir, 1989). La literatura señala que los roles de los sujetos, y específicamente de las mujeres, no solo estarían condicionados por el patriarcado 
como estructura dominante, en el que "la familia (...) es el principal foco del impacto de la cultura ambiental sobre la persona: sobre la personalidad; sobre la formación del ser humano, el productor-consumidor, el ciudadano, el hijo de Dios" (Fromm, Horkheimer y Parsons, 1986, p. 124), sino que además esta interviene en las etapas del ciclo vital del desarrollo humano. Para Berger y Luckmann (2001), a través de la socialización se produce la internalización "de la sociedad" (p. 196), lo cual es clave para la conformación de la identidad de las personas. Esto porque niñas y niños crecen creyendo que el mundo es solamente de una forma y que no existe otra, puesto que es el único modo que les enseñaron para entender la realidad. De esta manera, todo el sistema de creencias y de valores que las mujeres alcohólicas recibieron por parte de sus familias, escuelas, y amigos es realmente significativo para ellas y no está exento de la reproducción cultural que realizarán a lo largo de sus vidas.

En coherencia con lo anterior, la antropóloga feminista Marcela Lagarde (2005) emplea el concepto de "cautiverios" -que incluye los distintos ámbitos de la vida de las mujeres y la opresión del género que condiciona su pensar, sentir y actuar- para dar cuenta de la subordinación femenina. En esta investigación partimos del supuesto de que, tanto en el ámbito privado como en el público, existen elementos provenientes de la cultura heteronormativa que comprenden espacios físico-sociales en los que se desenvuelven las mujeres (la casa, el trabajo, grupo de amigos, familia, relaciones de pareja), y estos a su vez predeterminan las expectativas de género asociadas socioculturalmente a las mujeres en esos contextos.

Por otra parte, si bien el consumo de alcohol es una práctica asociada a lo masculino (Castillo y Gutiérrez, 2008) y aceptada socialmente como tal, las mujeres cada vez más se suman a esta práctica, debido, en parte, a los cambios en la división de roles. Paradójicamente, no por ello dejan de ser doblemente estigmatizadas, por ser mujeres y consumidoras de alcohol (Infonova, 2011; Freitas de Oliveira, Santos y Leal, 2007). El rechazo social que recae sobre las mujeres alcohólicas, así como en las adictas a 
otras drogas, suele ser más severo que para los hombres, principalmente porque, como ya fue señalado, dichos consumos afectan principalmente el desempeño de los roles de ama de casa y/o madre (Romo, 2005).

En el origen del consumo problemático de alcohol y en cómo esto afecta a las mujeres, se producen diferencias importantes pues, para Goffman (2006), se puede encontrar dos tipos de identidades; una de ellas es la virtual, cuyos atributos son adquiridos por su potencialidad, mientras que otra es la identidad social real, que se puede demostrar simplemente por su existencia. Sin embargo, ya sean atributos potenciales o realmente existentes, ambos pueden ser "profundamente desacreditador[es]", fundamentalmente porque es un lenguaje de relaciones y no de atributos" (p. 12).

Para Sau (1991), en la estructura patriarcal, sobre las mujeres recaen estigmas, ya que, debido a las características de sus cuerpos, les es imposible realizar labores que requieran uso de mayor fuerza y son consideradas como sujetos emocionales incapaces de tomar decisiones racionalmente (Montecino, 2006). Estos estigmas son propios del discurso heteronormativo y son naturalizados y aceptados socialmente; justamente, es esta aceptación del estigma "el rasgo central que caracteriza la situación vital del individuo estigmatizado" (Goffman, 2006, p. 19).

La estigmatización social es una de las consecuencias que recae sobre las mujeres que realizan conductas desviadas, es decir, se las estigmatiza por no cumplir con las expectativas del rol asignado y las normas sociales establecidas. Para profundizar en el concepto "conducta desviada" se utilizó la perspectiva de Becker (2014), con la cual se enfatizó cómo el consumo excesivo de alcohol, además de las consecuencias que se mencionaron, genera efectos colaterales que no estaban premeditados por las mujeres al momento del consumo, como, por ejemplo, ser una "outsider" o una "desviada". El autor define la desviación "como la infracción a algún tipo de norma acordada" (Becker, 2014, p. 28); es decir, las mujeres que "beben" deben tener ciertos límites al momento de enfrentarse al con- 
sumo, ya que las reglas sociales asociadas al género recaen sobre ellas independiente de los contextos en los cuales se encuentren y "deben comportarse como señoritas", "ser educadas", "respetuosas", etc. Pero, además, el hecho de abusar del consumo de alcohol les confiere lo que el autor denomina como "un nuevo estatus" (p. 51), y se les etiqueta como "locas", "curaditas", "borrachas", "adictas", entre otros adjetivos (Becker, 2014). Se generan prejuicios y estigmas sobre ellas tanto en estado de sobriedad como bajo los efectos del alcohol. Becker (2014) señala que "un rasgo desviado puede tener un valor simbólico generalizado, de forma tal que la gente presupone automáticamente que su poseedor también tiene otros rasgos indeseables asociados" (p. 52), lo que incrementa los problemas de tipo familiar, laboral, entre otros.

Según Becker (2014), "el último escalón en la carrera de un desviado es integrarse a un grupo desviado organizado" (p. 56), es decir, la persona puede integrarse a un grupo de desviados para intensificar el consumo o, para este caso en particular, las mujeres alcohólicas fueron capaces de verse sumidas en una adicción y buscaron ayuda profesional para tratarse en A.A.

\section{Marco metodológico}

Esta investigación se posicionó desde el paradigma interpretativo-fenomenológico, ya que se propuso identificar los elementos transversales a las historias de vida de las mujeres alcohólicas, detectar las expresiones de violencia hacia la mujer y, junto con ello, conocer los motivos por los cuales las mujeres acuden y permanecen en A. A.

El enfoque que se utilizó fue cualitativo, ya que, mediante el método biográfico de Bertaux asociado a las historias de vida, se explicó "las formas en que las personas en situaciones particulares comprenden, narran, actúan y manejan sus situaciones cotidianas" (Rodríguez, Gil y García, 1999, p. 33). Y también porque mediante la experiencia durante el trabajo de campo se construyó la investigación. 
La técnica utilizada fue la entrevista biográfica, cuyo instrumento de aplicación resultó ser un cuestionario construido bajo la modalidad de entrevista semiestructurada. Si bien el supuesto de investigación estaba determinado, se propuso dar libertad a los relatos y no inducir ciertas respuestas. Como estrategia, únicamente se preguntó acerca de ciertos aspectos claves, lo que permitió analizar posteriormente la información desde el método comparativo-comprensivo.

La unidad de análisis fueron mujeres alcohólicas que tuvieran más de cuarenta años (sobre la edad fértil) y que además estuvieran recuperadas de su adicción al alcohol pero que continuaran participando en A.A. (todas ellas miembros del grupo de A.A. de la Comuna de Providencia, Región Metropolitana, Chile). Esto con la finalidad de que pudiesen construir un relato de vida y de consumo a partir de la reflexión y el aprendizaje propios de los años. Es preciso mencionar que se realizaron tres sesiones por cada caso, con el objetivo de abordar en cada una de ellas una etapa determinada de sus vidas; de manera que en la primera sesión el tema fue la infancia, la segunda se enfocó en la adolescencia y juventud, y en la tercera se enfrentó la adultez y rehabilitación. Esta división permitió visualizar la socialización experimentada y la forma en que la estructura oprimió en cada ciclo vital a las mujeres. Asimismo, los relatos de las mujeres pueden no ser lineales o cronológicos, es decir, suelen existir saltos hacia adelante y/o hacia atrás en sus relatos de vida; además las entrevistas fueron semiestructuradas por lo que, ante cualquier duda o intención de profundización en cierta temática, las entrevistadoras no se guiaron por la pauta sino por su intuición investigativa.

Respecto del análisis y disposición de los resultados, las categorías con las que se trabajó fueron construidas tanto desde las revisiones bibliográficas como desde la información levantada previo a la realización de las entrevistas en las exploraciones de campo. 
Cuadro $\mathrm{N}^{\circ}$ 1: Unidad de análisis

\begin{tabular}{|l|l|l|}
\hline \multicolumn{2}{|l|}{ Mujeres alcohólicas centro de recuperación de A.A., comuna de Providencia. } \\
\hline Entrevistadas & Edad & Años en recuperación \\
\hline Mujer 1 & 54 & 15 \\
\hline Mujer 2 & 88 & 36 \\
\hline Mujer 3 & 45 & 5 \\
\hline
\end{tabular}

\section{Análisis de resultados}

I. Las maneras en que operan los elementos provenientes de la cultura patriarcal en mujeres alcohólicas: desde la familia, a través del género, y en los procesos de socialización

Teniendo en cuenta que los elementos provenientes de la cultura heteronormativa entregan las pautas del deber ser a las mujeres y que a partir de ellas se predetermina el correcto ejercicio de los roles impuestos, en los casos estudiados el inicio del consumo de alcohol se da en la infancia, principalmente por la insatisfacción de su desempeño en el deber ser femenino, al no considerarse para ellas mismas y para otros como atractivas o bonitas, y/o femeninas. Al llegar a la adolescencia el consumo de alcohol forma parte del relacionamiento para suplir la socialización deficiente, su utilización se desvirtúa pues es empleado para incrementar la autoestima, la seguridad, y/o sentirse considerada por los pares, y así enfrentar las distintas situaciones de sus vidas. En el relato de las mujeres se visualizó que, si bien el consumo comienza en el núcleo familiar y se potencia con las amistades durante la adolescencia, al relacionarse con parejas igualmente consumidoras se incrementa aún más y se produce un sentimiento de disconformidad con la propia vida y con el desempeño del rol.

...yo siempre fui una mujer tímida, que no me valorizaba, que era... insegura... cachay, entonces... yo era como muy chica, muy tonta, yo me acuerdo que todos mis amigos andaban pendientes de ellas, a mí no me inflaban, cachay, entonces como yo empecé a sentir esa sensación del alcohol, yo... en se minuto 
cuando estaba joven era, como que me sentía más simpática. (Mujer 1)

En palabras de Lagarde (2005), estas mujeres están cautivas de su género, ya que la estructura patriarcal determina expectativas respecto al género y, ante la presión de no lograr cumplir con ellas, las mujeres entrevistadas, mediante el consumo de alcohol y otras drogas, intentaron reforzar aspectos de la personalidad, como sentirse atractivas, simpáticas, parte de un grupo y obligándose incluso a mantener el deseo sexual hacia una persona, sin percatarse de que en dicho intento el consumo de alcohol se volvió problemático.

En cuanto a los aspectos normativos derivados del género, presentes en las trayectorias de consumo de estas mujeres, se visualiza que la estructura heteronormativa fue rígida en la familia de origen, principalmente respecto de las expectativas de sus apariencias físicas, y luego, en sus familias posteriores, esta fue dura, aunque no constante, especialmente en las experiencias sexuales y ante las exigencias de sus roles tanto de madres como de esposas. Es importante resaltar que estas mujeres pudieron tener control sobre su cuerpo al abortar en más de dos ocasiones cada una, pero dicho actuar estuvo subsumido en el rol de maternidad ya que, de no hacerlo, habrían sido madres irresponsables por no poseer los medios económicos para el bienestar del bebé o por la incapacidad de cuidarlo por su estado permanente de ebriedad.

Las implicaciones de lo heteronormativo en las trayectorias de vida de las mujeres se expresaron mediante su desempeño en la esfera pública y privada, desde una posición de desigualdad frente a los hombres (Dobash y Dobash, en Ferrer y Bosch, 2005), de manera que incluso el supuesto control de sus cuerpos respecto a la fertilidad no fue tal, pues en ello primó el ser buena madresposa. La situación económica de las familias de origen de las mujeres les permitió tener una infancia libre de labores del hogar; producto de ello, éstas no eran una obligación en el núcleo 
familiar. Tampoco se establecieron labores "propias del género de las mujeres" (Araya; Babcock, Costa, Green y Eckhardt, citados en Espinosa, 2010) que les hicieran experimentar machismo en este ámbito en particular durante la infancia.

Como señalan Berger y Luckmann (2001), la socialización primaria es fundamental en la inserción social de la persona. La socialización secundaria se asimila a la primaria, pero entrega otros conocimientos y aprendizajes de la vida social relacionados con los ciclos vitales que van viviendo las personas. Durante la adolescencia, estas mujeres se enamoran, asisten a fiestas con amigos y beben como una práctica habitual y normal; junto con ello, consumir cualquier tipo de alcohol genera en ellas sentimientos de seguridad, confianza en sí mismas, refuerza su ego y se sienten más seguras de su personalidad. El alcohol se transforma en una herramienta de doble funcionalidad: en primera instancia es utilizado como un desinhibidor que además provoca integración y aceptación social, y, en segunda, se convierte en un medio de evasión de la realidad.

El primer consumo de alcohol en estas mujeres se produjo entre los 6 y 7 años. El hecho de que estuviera presente desde la socialización primaria y familiar sugiere que fue en el núcleo familiar donde las mujeres iniciaron su experimentación con el alcohol y al momento de la adolescencia tuvieron menos prejuicios frente al consumo, lo que además se complementa con la independencia y autonomía que caracteriza a las tres mujeres entrevistadas. Algunas manifestaron que en fiestas familiares, como matrimonios y cumpleaños donde adultos estuvieron presentes, estos no se percataron de que las niñas habían bebido, o bien, los mismos adultos les dieron a probar alcohol sin medir las consecuencias de dicha acción. Dos de estas mujeres señalaron que sus familiares eran conscientes del consumo a esa edad. Una de ellas relató que se comía la fruta que quedaba en tragos preparados, y la otra que su abuelo producía licor de nuez y les daba de probar a ella y otros dos primos para que "aprendieran a beber". 
Las tres mujeres señalan que gustaron más de la sensación de beber alcohol que del sabor, lo que se proyectó en todas sus trayectorias de consumo posterior. Principalmente producía en ellas sensaciones de euforia, seguridad y confianza en sí mismas. Incluso una de ellas siempre se ofrecía a ayudar a la familia en los cumpleaños y festividades que se hicieran en su casa, para así poder beber a escondidas de la familia; en este tiempo ella tenía aproximadamente 14 años.

Sin embargo, los roles dentro de la familia posterior que ellas mismas conformaron cambiaron de forma significativa, pues las responsabilidades se incrementaron, al igual que la opresión del género femenino, ya que contraer matrimonio les dio la responsabilidad de mantener un hogar, un esposo, hijos y a sí mismas. Dentro de estas familias, el rol de madresposa influyó en la disconformidad respecto de su realidad y modos de vida, ya que se encontraban a disposición del cuidado de otros y no de sí mismas. Fue por esta disconformidad que se aproximaron al consumo de alcohol y que posteriormente desencadenó su alcoholismo, convirtiéndose este último en un medio para evadir la realidad.

\section{Expresiones de violencia}

Respecto de la experiencia sexual de las entrevistadas, dos de ellas señalaron iniciar su vida sexual a los 14 años. Una de ellas describe que desde muy pequeña sintió la voluntad incontrolable por tener sexo y que no recuerda la pérdida de su virginidad, porque en todas las relaciones sexuales que mantuvo durante la adolescencia estaba borracha. De manera que el alcohol era utilizado para evadir la responsabilidad de tener, por un lado, el deseo incontrolable, y, por otro, relaciones sexuales a corta edad. La segunda mujer entrevistada señaló que comenzó a tener relaciones sexuales con el pololo que posteriormente se convirtió en su marido a los 17 años. Cuando ya estaba casada tuvo relaciones sexuales con vecinos, pues estando bajo los efectos del alcohol se daba licencia para hacer lo que quisiera y de cierta forma excu- 
sar su comportamiento. Señaló que, además de consumir alcohol para evadir problemas, lo hacía para sentir deseo sexual por su marido. Por lo tanto, esta respuesta sugiere que el alcohol reencauza la conducta de la mujer (Agoff, 2006), haciendo que esta satisfaga a su pareja sin considerar lo violento de dicho acto. La tercera mujer no señaló haber tenido muchas parejas sexuales, tenía relaciones duraderas pero con personas drogadictas y alcohólicas, lo cual intensificó el consumo de alcohol.

En consecuencia, la opresión hacia las mujeres, derivada del género, respecto de las formas en que experimentan y viven su sexualidad, son igual de violentas que los cautiverios a los que hace referencia Lagarde (2005), pues el lugar de la procreación era otorgado únicamente a la madresposa, la satisfacción de los hombres era propio de la puta y la abstinencia sexual decisión de la monja. Cualquier otra forma en que la vida sexual de la mujer no estuviera a disposición de otro, en este caso de los hombres, ya sea por no brindarle hijos o por no saciar su deseo sexual, era una desviación de la norma y, en consecuencia, su incumplimiento como madresposa, puta o monja.

Respecto del rol de madresposa de las mujeres entrevistadas, todas abortaron de dos a tres veces entre los 15 y 23 años de edad. Ninguna manifestó arrepentimiento de haber abortado, sino que justificaron su actuar porque no contaban con los medios económicos ni de salud (por el alcoholismo) para mantener hijos en ese momento, por lo que sienten haber actuado en el momento preciso y con la mejor decisión. Dos de ellas señalaron que, al trabajar, podían solventar sus gastos; sin embargo, la mujer que dedicó la mayor parte de su vida a ser solo ama de casa no poseía ingresos económicos y dependía económicamente de su marido. A causa de dicha inestabilidad, al inicio de su matrimonio tomó la decisión de abortar tres veces, ya que su marido no le proveía el dinero suficiente para mantener un bebé y porque él le sugería que lo hiciera por el bienestar de la relación. En consecuencia, las mujeres entrevistadas se enfrentan a una situación de tensión entre ser independientes y cumplir 
con las expectativas del género, lo que en definitiva se convierte en una acción violenta hacia ellas, pues al oprimirlas mediante las expectativas de los roles de género, sus decisiones frente a la maternidad se encuentran condicionadas por ser "buena" o "mala" madre.

El intento de evasión de la realidad fue puesto en pausa por dos de las mujeres entrevistadas, ya que optaron por tener hijos durante su matrimonio, y si bien ambas dejaron de consumir alcohol en el periodo de gestación y lactancia, luego de ello continuaron bebiendo, ya que sus temores sobre la inestabilidad económica y pérdida de confianza en sí mismas las continuó induciendo a evadir la realidad (Romo, 2005). Una de las mujeres señaló que en el diario vivir consumía alcohol desde que sus hijas se iban al colegio, y que a causa de estar borracha todos los días de la semana y desde muy temprano en la mañana, no se bañaba, se le quemaba la ropa mientras la planchaba y era grosera para expresarse verbalmente. Otra de las mujeres dijo que, a pesar de la ausencia de afectividad hacia su hija, fue una madre responsable, pues resolvió todas sus necesidades materiales supliéndolas por las afectivas. La tercera mujer, que optó por no tener hijos, consideraba que no podía ser mamá porque era muy irresponsable, que no estaba dispuesta a frenar el consumo de alcohol y drogas ya que, al tener un hijo, aumentaría la culpa que ya sentía por el hecho de consumir.

Los tipos de relacionamiento que experimentaron las mujeres entrevistadas también estuvieron marcados por episodios de violencia. Por ello, es posible destacar lo que señala Infonova (2011) respecto de la conducta violenta, ya que en cualquiera de sus expresiones produce sujetos vulnerables, y que, a su vez, el consumo de drogas hace doblemente vulnerables a las mujeres, pues disminuye sus grados de autonomía, convirtiéndolas en posibles víctimas de maltrato. Por lo tanto, se examina cómo la estructura patriarcal castiga especialmente a las mujeres por medio de la violencia en sus diferentes expresiones cuando ellas no cumplen con la norma del género femenino. 
Una de las entrevistadas señaló que nunca sufrió violencia física en su vida; en cambio, dos de ellas sí sufrieron este tipo de violencia por parte de sus parejas, que se prolongó por años: estas fueron justamente las mujeres que decidieron casarse y tener hijos. Una de las mujeres golpeadas dijo que tampoco recuerda mucho estos episodios de violencia, porque el alcohol la tenía anestesiada y pasaba la mayor parte del tiempo bajo sus efectos.

En consecuencia, así como señala Infonova (2011), se produce un círculo vicioso al utilizar el consumo de drogas para la adaptación y mitigación del dolor psíquico producido por la violencia.

Respecto de la violencia psicológico-simbólica, se puede señalar que la primera entrevistada vivió desde los 4 hasta los 9 años de edad con una tía enferma; cuando regresó con sus padres y hermanos sintió el rechazo de estos por su sobrepeso. La segunda mujer entrevistada manifiesta que su mamá estaba empeñada en cambiarle el aspecto físico para que fuera más bonita; su marido se avergonzaba de ella al verla borracha y tirada en la cama, llena de vómito y feca producto de la ebriedad; la tercera mujer, al vivir la separación de los padres y verse sin ambiciones en la vida, se sentía frustrada por tomar repetidamente malas decisiones sin apoyo de su familia y eso la hacía consumir cada vez más.

$\mathrm{Al}$ enfrentarse a situaciones de estrés, bajo reconocimiento social, rechazo familiar, inestabilidad económica, burlas, presión y responsabilidad de ser madres, baja autoestima, pérdida de confianza en sí mismas, entre otras, las mujeres intentaban evadirlas mediante el consumo de alcohol y, al mismo tiempo, se mantenían en el círculo de violencia al no detener las situaciones de abuso. Además, consideramos el aborto parte de la violencia obstétrica producto de la realización de dicho acto en malas condiciones o en clandestinidad, haciendo que mujeres deban pasar por situaciones denigrantes por el carácter ilegal que se le atribuye. 


\section{Tipos y modos de consumo}

Respecto de los tipos y modos de consumo, y dentro de lo que determinamos como consumo independiente, fue importante en dos de estas mujeres comenzar a trabajar, ya que de ese modo podían solventar todos sus gastos, inclusive sus adicciones. El trabajo remunerado permite a las mujeres sentirse independientes. Una de ellas señala que compraba y consumía alcohol todos los fines de semana con su pareja, y la otra, al vivir sola, bebía todos los días mezclando el alcohol con otras drogas, como crack, cocaína, marihuana, hongos alucinógenos, LSD y otros.

La segunda mujer no trabajaba, ya que no pudo terminar la universidad y al casarse se dedicó a ser ama de casa, lo que no impidió que consumiera alcohol de forma desmedida. En su relato señala que vendió cosas personales para poder comprar alcohol, y que cuando su alcoholismo se agravó comenzó a comprar alcohol para heridas en farmacias, puesto que este era más efectivo para el deseado estado de ebriedad.

Si bien el consumo de alcohol se inicia en el seno familiar, estas mujeres tienen conciencia de la influencia que cumplieron sus parejas en el acercamiento e incremento del consumo, y que posterior a estas relaciones amorosas comenzaron a consumir en cualquier contexto. Esto se relaciona con lo señalado por Infonova (2011), pues las mujeres no solo consumen drogas con sus parejas consumidoras a causa de curiosidad por nuevas experiencias, sino que para sentirse más identificadas con sus parejas hombres. La primera entrevistada consumió de manera progresiva durante una relación amorosa, ya que comenzó a ser parte del círculo de amistades de su pareja, quienes bebían en exceso, generando además una relación amorosa basada en la violencia y consumo problemático incluso después de estar casados.

En las mujeres entrevistadas, el consumo recurrente comienza entre los 13 y 14 años, su principal compañía durante las fiestas son los amigos, con quienes comienzan a transformar el consumo, y dos de ellas, a experimentar con otras drogas, prin- 
cipalmente marihuana y pastillas. En la adultez mantuvieron un consumo social de alcohol, es decir, lo compartían con sus amigos después del trabajo o los fines de semana. A medida que avanzó el tiempo, las entrevistadas expresan que se agudizó el consumo y con ello la dependencia al alcohol, y comenzaron a beber solas en sus casas, en cualquier día y a cualquier hora.

El consumo problemático de alcohol de las mujeres entrevistadas se convirtió en una práctica inadecuada para sus amistades y para sí mismas, ante los prejuicios que conlleva ser bebedora incluso en estado de sobriedad (Becker, 2014). En consecuencia, la opresión de las expectativas del género produjo, por un lado, que las mujeres se aislaran de sus círculos de amistades e intentaran ocultarlo principalmente de sus familias.

IV. Motivos por los cuales las mujeres alcohólicas acudieron y permanecen en A.A.

De la categoría que se refiere al ingreso y permanencia de las mujeres entrevistadas en la comunidad de A.A. se desprenden motivos y causas (Agoff, 2006). Los motivos los abordaremos como aquellas acciones guiadas por la conciencia que buscan un objetivo determinado; mientras que las causas se refieren a los componentes externos que condicionan el actuar de la mujer. Dentro de los principales motivos por los cuales estas mujeres acudieron a A.A. se encuentra la influencia de familiares, tanto por el bienestar de las mujeres como por el cuestionamiento de su rol de madres.

Sin embargo, durante la adultez y participando en A.A. estas mujeres realizan un trabajo reparatorio, pues en la interacción de grupo con personas que comparten experiencias comunes derivadas del alcoholismo generan lazos de confianza y de amistad. En consecuencia, permanecer en la comunidad les brinda estabilidad física, psicológica y emocional, pero a partir del trabajo espiritual, pues dejan en la voluntad de un ser superior (Dios) su recuperación, eximiéndose de culpabilidad y de las consecuencias de sus actos pasados cuando aún no podían abandonar el alcohol. 
Las entrevistadas tenían entre 45 y 88 años de edad al momento de realizar las entrevistas. Para Agoff (2006), las mujeres mayores de 40 años poseen conciencia crítica de su realidad, entendiendo que posiblemente desde las mujeres alcohólicas nació la motivación para realizar el proceso de recuperación. Sin embargo, solo una de las tres manifestó interés de recuperarse por cuenta propia, debido a la preocupante situación tanto de salud como social en la que se encontraba; junto con ello señaló que no se sentía feliz en su vida por lo que decidió pedir ayuda. Cabe destacar que esta entrevistada es la que no se casó ni tuvo hijos, ya que la recuperación de las otras dos mujeres estuvo condicionada por la presencia de los hijos; para la segunda mujer entrevistada, fueron ellos, junto con su yerno, quienes sugirieron la recuperación. Para la primera entrevistada fue la posible pérdida de tuición de su hija, luego de ser amenazada por sus hermanos y su madre.

Yo, cuando pedí ayuda, era porque llevaba dos meses sin pagar arriendo, no pagaba por usar ese dinero en el consumo. Nunca tuve nada para vender.

Yo cuando le pedí ayuda a mis papás para venirme, agarré mis cosas, mi uniforme de trabajo y el pijama, no tenía más que eso... porque dejé de comprarme o porque subí más de peso y no me compré. (Mujer 3)

Mi hermano me dijo 'nosotros contigo no podemos hacer nada, así que la niña va a vivir con nosotros...' y ahí yo ya tomé conciencia y dije yo, 'ya, hay que buscar ayuda...' así que llegué a alcohólicos anónimos. (Mujer 1)

En los relatos de las entrevistadas que fueron madres no aparecen amistades significativas en el inicio del proceso de recuperación; sin embargo, la tercera mujer entrevistada, que decidió rehabilitarse por cuenta propia, se contactó con un amigo ya rehabilitado, quien le recomendó A.A. Las tres entrevistadas señalan que en A.A. se sintieron escuchadas, acogidas y queridas, además de comprendidas, puesto que en ello reside la importancia de seguir siendo parte de la comunidad. 
El alcoholismo es una enfermedad que no tiene cura; la única forma de recuperarse es absteniéndose de beber alcohol; siendo esto parte fundamental de los motivos por los cuales las mujeres entrevistadas permanecen en la comunidad, ya que al superar los doce pasos ellas se encuentran en condiciones adecuadas para ayudar a otros alcohólicos en su proceso de recuperación. Al ser parte de A.A. se ejercen dos roles: por un lado, ser quien necesita ayuda con su enfermedad y, por otro, quien ayuda al enfermo compartiendo sus experiencias de vida.

\section{Conclusiones}

Los resultados obtenidos de esta investigación han permitido visualizar lo normativo de la estructura patriarcal hacia el género femenino. Es decir, la manera en que regula el comportamiento social y privado de este, entorpeciendo incluso el inicio del proceso de su recuperación. Por ello, fue posible profundizar en los elementos provenientes de la cultura patriarcal presentes en las trayectorias de consumo de alcohol en mujeres alcohólicas.

Mediante las entrevistas realizadas se visualiza cómo el consumo de alcohol se presenta en las mujeres entrevistadas desde temprana edad. De ello lo más significativo es que no consumían por el sabor de la bebida alcohólica, sino más bien por los efectos que producía en ellas; de manera que las sensaciones -principalmente de inseguridad y de baja autoestima- desaparecían en la ebriedad, lo que responde a la conducta evasiva de las mujeres por el sentimiento de disconformidad con sus vidas y con las expectativas del rol femenino. En consecuencia, y desde su propio relato, se evidenció que el consumo de alcohol era una práctica para evadir su realidad, de manera que esto conllevó al aumento progresivo del consumo de alcohol, que desencadenó en alcoholismo.

A partir de los resultados obtenidos se puede afirmar, mediante los tres relatos, que existen elementos provenientes de la cultura patriarcal comunes en la trayectoria del consumo de 
alcohol en mujeres, y estos se dan de forma transversal, independientemente del nivel socioeconómico y de estudios de las mujeres (Romo, 2005). Además, las mujeres consumidoras de alcohol sufren en gran medida discriminación, estigmatización y presión social (y/o familiar), producto del rol que se les atribuye respecto del género dentro del núcleo familiar. De este modo, acuden principalmente a A.A. por decisión propia, familiar o por posible pérdida de la tuición de los hijos. Al ser transversal el consumo problemático de alcohol en mujeres, dicha práctica está condicionada a situaciones como lazos familiares deteriorados a temprana edad, violencia intrafamiliar en distintas etapas de su vida, y además motivada por un sentimiento de aislamiento social, lo cual se ve reflejado en los problemas de visualización del consumo problemático y a la hora de pedir ayuda.

Al sentar las bases de los roles de género, la estructura heteronormativa oprime al género femenino, del cual se espera el cumplimiento de expectativas de rol a partir de la socialización con otros. Esto delimita el actuar de las mujeres, las cuales, al no estar satisfechas con su desempeño respecto a las expectativas del rol, realizan conductas desviadas: una de ellas, el consumo problemático de alcohol para evadir la realidad. Esta práctica las estigmatiza y reprime, teniendo como consecuencia un doble castigo para ellas: por ser mujeres y por ser alcohólicas, reflejándose en la tardanza tanto de la visualización del consumo problemático como del inicio de la recuperación.

Sin intencionar los datos para que el supuesto fuera aceptado, por medio de la revisión bibliográfica y por las entrevistas efectuadas, se puede señalar que el problema investigado es mucho más complejo, puesto que la estructura social dominante es problemática, diversa y no deja nada al azar, ya que repercute constantemente en cada ámbito de la vida de los hombres y de las mujeres, donde estas son las más perjudicadas porque viven en una posición de inferioridad constante, condicionada por el género atribuido socioculturalmente a partir de su sexo. Solo por el hecho de ser mujeres son juzgadas y estigmatizadas por su 
condición de alcohólicas, sus roles se ven cuestionados porque se espera que la mujer sea un pilar fundamental en la familia: es quien está a cargo de la crianza y el cuidado de los hijos y del marido, por ello su figura no puede verse cuestionada, tal como se ha señalado (Romo 2005; Infonova 2011; Freitas de Oliveira, Santos y Leal, 2007). Asimismo, la familia, al ocultar cualquier indicio de alcoholismo en alguna de las mujeres del hogar (abuela, madre, hija, tía, etc.), se convierte en cómplice de este alcoholismo y del retraso en la recuperación de la mujer. El alcoholismo es una condición que no tiene directa relación con la pertenencia a estrato socioeconómico, nivel educacional o cultural: el alcoholismo puede afectar a cualquier persona que se sienta disconforme con su vida y con el desempeño de sus roles de género, puesto que el no cumplimiento de la norma y/o del deber ser es castigado socialmente.

El principal aporte de esta investigación al campo de la sociología comprensiva y del género es que se trata de una investigación cualitativa que, a través de la fenomenología, busca conocer las experiencias subjetivas de las mujeres alcohólicas visualizando la problemática del alcoholismo desde la perspectiva de género. De ese modo se logra entender cómo las mujeres experimentan el mundo social a través de sus vivencias, intentando explicar los significados y las definiciones que ellas otorgan a sus trayectorias de vida, para conocer una problemática como el alcoholismo desde la perspectiva de género.

Es preciso realizar más investigaciones que visualicen la problemática de mujeres alcohólicas, ya que éstas traen consigo un sinnúmero de antecedentes que reflejan la transversalidad de los problemas sociales en la actualidad, a raíz de la estructura heteronormativa y su incidencia en los distintos ámbitos de la vida, desfavoreciendo en todos ellos a las mujeres; un ejemplo es el consumo de alcohol en la infancia, vínculos familiares quebrantados, violencia de género hacia la mujer en sus distintas expresiones, aislamiento social, entre otros. Por ello, se precisan investigaciones que aborden la recuperación de las mujeres alcohólicas con 
un carácter de reinserción social, mediante la formación de redes y herramientas de desarrollo personal. También es importante considerar los resultados de esta y otras investigaciones, para que se conviertan en un aporte en la conformación de políticas públicas, además de responder a las necesidades de centros de recuperación (públicos y privados) para mujeres, focalizando la atención en mejoras en la aplicación de los tratamientos, puesto que al enfrentarse a la realidad de una mujer alcohólica se deben enfrentar también miedos, traumas, violencia, opresión, baja autoestima y una disconformidad con el rol y, en general, con la propia vida.

\section{Referencias}

Agoff, C., Rajsbaum, A. y Herrera, C. (2006). Perspectiva de las mujeres maltratadas sobre la violencia de pareja en México. Salud Pública de México, 48(2), 307-314. Recuperado el 26 de junio de 2016 de http://www.redalyc.org/articulo. oa? id=10604811

Alcohólicos Anónimos (2016). Método de recuperación. Recuperado el 5 de agosto de 2016 de http://www.alcoholicosanonimoschile.cl/metododerecuperacion.html

Beauvoir, S. (1989). El segundo sexo: La experiencia vivida. Buenos Aires: Siglo Veinte.

Becker, H. (2014). Outsider: hacia una sociología de la desviación. Buenos Aires: Siglo Veintiuno Editores.

Berger, p. y Luckmann, T. (2001). La construcción social de la realidad. Buenos Aires: Amorrortu Editores.

Cáceres, D. (2016). Sobre la semántica del femicidio en Chile. Sociedad y Economía, 31, 239-262. Recuperado el 5 de agosto de 2016 de http:/ /www.redalyc.org/pdf/996/99647007011.pdf

Castells, M. (2000). El fin del patriarcado: movimientos sociales, familia y sexualidad en la era de la información. En M. Castells, (Ed.), La era de la información (pp. 159-269). México: Siglo XXI Editores.

Castillo, p. y Gutiérrez, A. (2008). Consumo de drogas en mujeres asistentes a centros de tratamiento especializado en la Ciudad 
de México. Salud Mental, 31(5), 351-359. Recuperado el 26 de junio de 2016 de http://www.scielo.org.mx/scielo. php?script=sci_arttext\&amp;pid=S018533252008000500003

Cobo, R. (2009). Otro recorrido por las ciencias sociales: género y teoría crítica. En M. Aparicio, B. Leyra y R. Ortega (Eds.), Cuadernos de género: políticas y acciones de género (pp. 11-52). Madrid: Universidad Complutense.

Colimil, F. y Ramírez, D. (2016). Soy adicta y mi droga preferida es el alcohol: la opresión del género sobre las trayectorias de vida de mujeres alcohólicas (Tesis de pregrado). UCSH, Santiago.

DIANOVA (2011). Violencia de género, alcohol y drogas. Infonova (22), 01-12. Recuperado el 26 de junio de 2016 de http:/ /www. dianova.es/images/pdf/publicaciones/Infonova/Infonova_22.pdf

Espinosa, J. (2010). Expresión de la violencia en parejas con consumo de alcohol. Psicología y Salud, 20(2), 221-229. Recuperado el 26 de junio de 2016 de http:/ /www.uv.mx/psicysalud/ psicysalud-20-2/20-2/Jaime-Espinosa-Cotto.pdf

Ferrer, V. y Bosch, E. (2005). Introduciendo la perspectiva de género en la investigación psicológica sobre violencia de género. Anales de Psicología, 21(1), 1-10. Recuperado el 26 de junio de 2016 de www.um.es/analesps

Freitas de Oliveira, J., Santos, M. y Leal, C. (2007). The interference of the care context with the visibility of the drug consumption by women. Latino-am Enfermagem, 15, 247-252. Recuperado el 26 de junio de 2016 de http:/ /www.scielo.br/scielo. php?script=sci_arttext\&pid=S0104-11692007000200009

Fromm, E., Horkheimer, M. y Parsons, T. (1986). La familia. Barcelona: Ediciones Península.

Goffman, E. (2006). Estigma. Buenos Aires: Amorrortu.

Lagarde, M. (2005). Los cautiverios de las mujeres: madresposas, monjas, putas, presas y locas. Ciudad de México: Universidad Nacional Autónoma de México.

Montecino, S. (2006). Madres y huachos: alegorías del mestizaje chileno. Santiago: Catalonia.

Rodríguez, G., Gil, J. y García, E. (1999). Metodología de la investigación cualitativa. Málaga: Aljibe.

Romo, N. (2005). Género y uso de drogas: la invisibilidad de las mujeres. 
Monografías Humanitas, 5, 69-83. Recuperado el 26 de junio de 2016 de http:/ / digibug.ugr.es/bitstream/10481/22315/1/ g\%C3\%A9nero \%20y $\% 20$ uso $\% 20$ de $\% 20$ drogas $\% 2 c \% 20$ la\%20invisibilidad.pdf

Sánchez, L. (2008). Guía informativa de género y drogas. Recuperado el 5 de agosto de 2016 de https://dialnet.unirioja.es/servlet/ libro? codigo $=514033$

Sau, V. (1991). Ética de la maternidad. Recuperado el 5 de agosto de 2016 de http://www.ub.edu/SIMS/pdf/MujeresSociedad/ MujeresSociedad-13.pdf

SENDA (2016). Funciones. Recuperado el 26 de junio de 2016 de http:/ / www.senda.gob.cl/quienes-somos/funciones/

Vieytes, R. (2004). Metodología de la investigación en organizaciones, mercado y sociedad. Buenos Aires: De las Ciencias. 